binocular vision in the sense of identical functioning of the two eyes does not exist. Walls ${ }^{7}$ has recently suggested an explanation of eye-dominance by what he termed his theory of directional dominance, claiming that the record of the innervations to the muscles of one eye only are used for the construction of binocular percepts of visual direction. This does not mean that the other eye is unused, but merely implies a differentiation in function of the two eyes. Walls mentioned the fact that after practice the microscopist is able to keep both eyes open without distracting his attention from the slide. If he should, however, attempt to use the other eye, the nondominant, he will find that the contents of the field of view of the dominant eye are seen superimposed on the instrumental field, and seem to interfere with good observation of the slide. It appears that the benefit of eye-dominance is that it clears the visual field by giving the right of way to the image of one eye, the dominant, making it appear more substantial than the other, which tends to be suppressed.

This survey of the commonest types of eyedness and some interpretation of the phenomenon reveals at least the range of factors considered important by different investigators, and also the necessity for caution in the acceptance of the findings based on such tests. In spite of this, it must be maintained that the dominant eye is a reality, though some of the statements on the subject have been rash, and many of the findings of dubious validity.

[May 16.

'Gould, G. M., "Righthandedness and Lefthandedness" (Lippincott, 1908).

"Parson, B. S., "Juefthandedness-A New Interpretation" (Macmillan Co., 1924)'

${ }^{2}$ Gahagan, L., J. Gen. Psychol., 9, 455 (1933).

' Jasper, H. H., and Rancy, E. T., Amer. J. Psychol., 49, 450 (1937).

' Crider, B., Child Development, 6, No. 2, 163 (1935).

- Lehman, H. C., and Webb, F. E., Motor Skills Research Exchange, 3, No. 1 (1951)

"Walls, G. L., A.M.A. Arch. Ophthalmol., 45, No. 4, 387 (1951).

\section{RADIATION CHEMISTRY}

$\mathrm{T}$ HE Faraday Society held a general discussion on "Radiation Chemistry" in the University of Leeds during April 8-10. The meetings were well attended and there were many visitors from overseas -forty-four from fourteen different countriesincluding many well-known workers in this field, among whom was Prof. K. F. Bonhoeffer, president of the Deutsche Bunsen-Gesellschaft für physikalische Chemie. The president of the Society, Sir Charles Goodeve, took the chair on the first day; on subsequent days Profs. C. E. H. Bawn and H. J. Emelćus presided. The authors were allowed five minutes to make the salient points of their contributions, so that ample time was left for discussion.

Radiation chemistry-the study of the chemical effects of photons, electrons, or positive ions of an energy greater than $50 \mathrm{eV}$. - has made rapid progress during the past few years, mainly owing to our increased knowledge of the chemistry of free radicals and of molecular ions. Its relatively slow development compared with that of photochemistry is due to the greater complexity of the primary process : in photochemical reactions this is simple and involves the absorption of a quantum of radiation by a molecule; but the transfer of energy from short wave-length radiation or from bombarding particles is complex, and prediction of the mechanism or of the products is uncertain. The object of this discussion, the first to be held on the subject by the Faraday Society, was to review the present position, to attempt a synthesis of the differing points of view of physicists, chemists and biologists, and to suggest further lines of development.

The general introduction was given by Prof. F. S. Dainton (Leeds), who reviewed the present difficulties of the subject and directed attention to the more important problems which have to be solved. He emphasized the lack of knowledge of the nature of the primary process ; the value of the energy $(W)$ required for the formation of an ion pair (formed along or near the track of the bombarding particle) is not known for liquids, nor how it is related to the ionization potentials of molecules with which the impinging particle collides. The relation of the mean ion density to the energy, mass and atomic number of the particles is, however, known qualitatively at least, and forms the basis of hypotheses which rolate the amount and nature of the reaction product with the mass and energy of the particle. Caution must be observed in applying to liquid systems conclusions based on the gaseous phase; but cases are known in which such extrapolations are legitimate. In polar media, however, the magnitudes of solvation energies and entropies are such as to invalidate the assumption of parallel behaviour in the liquid and the vapour, and they refer to equilibrium conditions not relevant in systems in which the charge transfer processes are very rapid. Prof. Dainton directed attention to some important questions which would be discussed at the meeting : the observed value of $G_{R}$, the fraction of the absorbed energy used in the formation of radicals, may be a function of the solute and may depend on other competing processes, and thus the maximum observed values are likely to be the more significant; differences in track density leading to variations in ionic yield may arise from quite small changes of energy - a point of considerable importance to radiobiologists; although the initial distribution of primary products is non-uniform, rate expressions based on uniform distribution may successfully account for the observed kinetic behaviour; the radiolysis of water produces species which may lead either to the oxidation or the reduction of certain solutes, and a considerable knowledge of the behaviour of the system under varying conditions is necessary before theoretical conclusions are made; the concept of protection and sensitization in biological systems may be of importance in simpler systems.

The first three papers were on the primary act. Prof. F. W. Spiers (Leeds) reviewed the processes of absorption of ionizing radiations in terms of the quantum energy, and discussed the relative importance of the photoelectric, Compton recoil and the pair-production processes in the absorption coefficient of water. (Data for water for some commonly used radiations were given.) The loss of energy by charged particles was discussed in terms of the stopping power of the medium given by the theory of Bethe and its modifications. The magnitude of the energy $(W)$ required to produce an ion pair was discussed in relation to the type of particle, its speed and the properties of the absorbing medium, and finally some indication was given of the distribution of ion pairs along the track of the fast-moving particle. Prof. H. S. W. Massey (London) dealt with the nature of gaseous ions, and the formation of clusters by alkali metal ions with polar molecules and with rare gas 
atoms, and described recent work on the molecular ions of the rare gases. He directed attention to certain features of atomic and molecular negative ions and to metastable ionic states. The second part of his paper was concerned with the rates of reactions involving ions, their classification, and the different processes which may occur. Prof. J. L. Magee (Notre Dame) examined the mechanism of charge neutralization in reactions between positive and negative ions by means of potential energy curves and their crossing-points, and gave a theory of the probability of transition. He concluded that the reactants retain their constituent atoms and configurations, and that dissociation may follow, but not rearrangements involving both the ions; highly excited states are to be expected, and radicals should be common reaction products. In the discussion which followed, some consideration was given to the problem of light emission and its role (if any) in radiation chemistry.

The next group of papers were devoted to actinometry. Dr. N. Miller and J. Wilkinson (Edinburgh) compared and criticized a number of systems which have recently been proposed for chemical dosimetry. They discussed the characteristics of suitable reactions in aqueous media, and summarized the pertinent data for the oxidation of ferrous ions in air-saturated $0.8 \mathrm{~N}$ sulphuric acid solutions, the reduction of ceric ions in similar solutions, and the hydroxylation of benzene in air-saturated water. There is considerable lack of agreement, except for ferrous sulphate solutions at lower dose-rates. The results were presented of their exhaustive tests designed to examine the variables which might cause irreproducibility in this system with $X$-and with $\gamma$-rays. The system is also applicable to electron dosimetry, but not to that of heavy particles. Non-aqueous systems were also discussed. Prof. F. S. Dainton, in his introductory remarks, expressed the gratitude of the meeting to Dr. Miller and Mr. Wilkinson, and hoped that it would be generally agreed to use the aerated ferrous sulphate actinometer for $\mathrm{X}$ - and $\gamma$-rays with doserates less than 1,000 r./min. Prof. Milton Burton (Notre Dame) strongly supported this view, and stressed the desirability of recording the total amount of chemical change per unit of energy absorbed (for example, as the so-called $G$-value or radiation yield per $100 \mathrm{eV}$.) rather than the ionic yield. J. Wright (Harwell) described experiments on the pile irradiation of various chemical systems, designed so that they could be related to calorimetric measurements, which will ultimately be the basis of dosimetry for pile radiation chemistry. It was found that the ferrous sulphate system was suitable for short irradiations and gave information about the $\gamma$-radiation resulting from the decay of fission products. General discussion followed, devoted partly to a consideration of postirradiation effects in the ferrous sulphate system.

Attention was then directed to the radiolysis of pure liquids. Prof. Burton and S. Gordon (Notre Dame) discussed the use of mass-spectrometric and radiation-chemical data on the same compounds for the interpretation of their mechanism of decomposition ; the ions revealed by the former process are complementary to the radicals of the latter: The application of these methods to the radiolysis of benzene and heavy benzene $\left(\mathrm{C}_{6} \mathrm{D}_{6}\right)$ shows that decomposition may occur either by bond rupture or by rearrangement; in a mixture the two compounds protect each other against radiation. A. PrevostBernas, A. Chapiro, C. Cousin, V. Landler and M. Magat (Paris) reviewed the possibilities and the limitations of two methods they have used for the determination of the number of free radicals formed in organic liquids by ionizing radiations, and gave estimates of the fraction of the energy used in free radical formation. They also discussed the mechanism of the production of free radicals in liquids irradiated in a pile, and the contributions of the different. radiations present.

The radiolysis of water is important not only for its own sake but also because it is a factor in the study of the radiation chemistry of aqueous solutions. Dr. A. O. Allen (New York) discussed his mechanism of the decomposition of water, based on the very large amount of experimental work done in his laboratories during the past five years, according to which molecular hydrogen and hydrogen peroxide are formed simultaneously with the production of hydrogen atoms and hydroxyl radicals, the molecular yield increasing, and the radical yield decreasing, with the ionization density of the radiation. The reverse reaction, $\mathrm{H}_{2}+\mathrm{H}_{2} \mathrm{O}_{2} \rightarrow 2 \mathrm{H}_{2} \mathrm{O}$, which is initiated by free radicals, is favoured by dissolved hydrogen and retarded by dissolved oxygen or hydrogen peroxide. The molecular decomposition is ascribed to reactions occurring in very small regions - hot spots. Dr. Allen also discussed the conditions to be satisfied in experiments designed to determine the molecular $\left(G_{M}\right)$ and radical $\left(G_{R}\right)$ radiation yields in water, and gave provisional values for these quantities which were the subject of some discussion. Prof. P. Bonet-Maury (Paris) also discussed the formation of hydrogen peroxide and of hydrogen from pure water by radiations of different types, and the possible secondary reactions and equilibria which may occur.

The next group of papers was on' the radiation chemistry of aqueous solutions. Dr. M. Haissinsky (Paris) considered the mechanism in dilute solutions in terms of the oxygenated radiolytic derivatives of water. Oxidation reactions preponderate; but reduction reactions are caused by hydroxyl radicals either directly, or indirectly by reaction with molecular hydrogen to give water and hydrogen atoms. It was suggested that hydrogen is produced chiefly by the process, $\mathrm{H}_{2} \mathrm{O}+e=\mathrm{H}_{2}+\mathrm{O}^{-}$, which leads to the formation of two hydroxyl radicals and one molecule of hydrogen per ion pair. Dr. C. B. Amphlett (Harwell) described experiments in which the oxidation of ferrous ions by $\mathrm{X}$ - and $\gamma$-radiation was studied in dilute sulphuric acid solutions of different concentrations and in the presence and absence of complexing agents. The variation in the initial oxidation-yield with $p \mathrm{H}$ suggests that the primary act is more complex than is usually assumed. Values of the 'equivalent redox potential' of irradiated water with respect to the ferrous-ferric system were obtained. Dr. W. M. Garrison and Prof. G. K. Rollefson (Berkeley) described elegant tracer experiments on aqueous solutions containing ferrous ion and carbon-14 dioxide irradiated with high-energy helium atoms, and found that the principal products were ferric ion and hydrogen in the molar ratio two to one; formic acid and much smaller amounts of formaldehyde and oxalate were produced. A mechanism giving a rate law which agrees with the experimental data was proposed.

Papers mainly concerned with the decomposition of hydrogen peroxide followed. Dr. J. Weiss (Newcastle upon Tyne) discussed the photochemical decomposition in relation to that by ionizing radiations, and obtained rate expressions for the reactions 
occurring within, and between, the tracks. Dr. E. J. Hart and M. S. Matheson (Chicago) described experiments with continuous and intermittent $\gamma$-radiation on aqueous hydrogen peroxide solutions in paraffin-coated cells, in which they found that the decomposition yield depended on the square root of the hydrogen peroxide concentration and inversely on the square root of the dosage-rate. A novel feature of the mechanism proposed was that chain termination results from the termolecular reaction $2 \mathrm{HO}_{2} \rightarrow$ $\mathrm{H}_{2} \mathrm{O}_{2}+\mathrm{O}_{2}$, in which hydrogen peroxide is regarded as being a third body of exceptionally high efficiency. Dr. M. Ebert and J. W. Boag (London) reported experiments on the decomposition of water and hydrogen peroxide by electrons and $\mathrm{X}$-rays, showing that with neutral water there is no dependence on the intensity of the radiation, but that in acid solution the equilibrium concentration of hydrogen peroxide produced by large doses depends on the intensity. The mechanism proposed received some confirmation by further work on hydrogen peroxide produced in neutral aerated water by 30 and $220 \mathrm{kV}$. X-rays and $1 \cdot 2 \mathrm{MeV}$. electrons by these workers in collaboration with Dr. M. Lefort (Paris and Leeds), H. C. Sutton (Leeds) and M. Ebert and Tikvah Alper (Hammersmith) using different analytical techniques. Dr. T. J. Hardwick (Chalk River) showed that, in the indirect action of $\mathrm{X}$ - and $\gamma$-radiation on aqueous ferrous and ceric sulphates, the yield varies with the initial energy of the ionizing electrons, and that this effect may be explained on the basis of existing theories of the primary action of ionizing radiation on water. The probability of the recombination of hydrogen atoms and of hydroxyl radicals was calculated as a function of the instantaneous electron energy.

Dr. E. Collinson and Prof. Dainton (Leeds) showed that the kinetics of the polymerization of aqueous acrylonitrile solutions induced by $X$ - and $\gamma$-rays are strongly different from photochemical or catalysed reactions, and are consistent with an inhomogeneous distribution of the radicals formed from water, while at higher dose-rates an increasing degree of homogeneity is indicated. The three types of radiation used produced the same results. In heavy water $\left(\mathrm{D}_{2} \mathrm{O}\right)$ solution irradiation leads to the formation of deuterium atoms which participate in the polymerization. In the discussion it was suggested that the unusual kinetics might in part be due to the insolubility of polymer in monomer.

The remaining papers dealt with more complex systems : Dr. G. Stein (Jerusalem) described the reactions of certain dyes in aqueous and non-aqueous systems, drew comparisons with biological systems and then discussed the role of the hydroxyl radical, free electrons and molecular oxygen in the radiation chemistry of organic substrates. Tikvah Alper (Hammersmith) reported experiments on the indirect inactivation of bacteriophage by ionizing radiations, and gave survival curves under various conditions. Dr. W. M. Dale (Manchester) discussed the possible protective effect of a second solute which may act as a competitive acceptor of free radicals, and the use of this effect, which is specific, as a measure of the protective power of various solutes. Dr. C. B. Allsopp and J. Wilson (London) showed that the quantity of indole decomposed in aqueous solution by $\mathrm{X}$-rays increases with increasing concentration of indole and with decreasing dosage-rate. The radiation and the photochemical decompositions follow similar courses. Dr. W. Minder and H. Heydrich (Berne) discussed the results of their experiments on the irradiation of halogenated hydrocarbons in organic solvents, which show that the formation of halogen acids depends on the dose, the concentration of the solution, the number of halogen atoms in the compound and the type of binding.

The meeting was very successful on both the scientific and social sides, and may fulfil the hope expressed by Sir Charles Goodeve at the guest-night dinner that it would stimulate the development of radiation chemistry as much as a previous discussion many years ago had that of the closely related subject of photochemistry. The papers, together with discussion on them submitted in writing to the secretary, will appear later as a separate publication of the Faraday Society.

J. W. Belton

\section{SCIENTIFIC EDUCATION OF PHYSICISTS}

$T$

HE pamphlet "The Scientific Education of Physicists"*, recently issued by the Institute of Physics, is a report prepared by the Education Committee of the Institute which should act as a useful guide, for students who wish to enter the profession of physics and for those who have the responsibility of advising or teaching such students, to the facilities available for the education and training of physicists.

The Institute recognizes only two qualifications as satisfying completely the academic requirements for election to corporate membership-an approved degree from a recognized university, and the Institute's own graduateship examination-although partial exemption from the graduateship examination may be claimed by those who have previously obtained the Higher National Certificate in applied physics. In addition to the suitable degree courses at university institutions, the report refers to the several technical colleges which provide advanced courses in physics, usually for the external degree of the University of London, but it warns the intending student that very often such technical colleges, with the exception of the London Polytechnics and a few of the main provincial colleges, have poor facilities for teaching physics and very rarely do their resources extend to an honours course in physics. Moreover, since he will find it extremely difficult to obtain adequate financial assistance at a technical college, for the student who wishes to continue and do postgraduate research, usually leading to the M.Sc. or Ph.D. degrees, it is almost essential that he should study at a university institution. However, many of the major technical, as well as university, Institutions do run excellent part-time courses, series of lectures and summer schools on advanced topies in physics which practising physicists will find of great value.

The report emphasizes the importance to the science student of an ability to express himself clearly, concisely and easily in English, both in speech and in writing. Recent discussion in the press has not settled whether the admitted prevalence of a low standard in English among physics students is due to lack of attention at the school or at higher levels; but in the report it is attributed mainly to individual negligence. The report concludes with appendixes giving factual data relating to the individual physics departments of all the universities and university * The Scientific Education of Physicists. Pp. 32. (London : Institute of Physics, 1952.) 28 . 